Pacific Journal of Mathematics

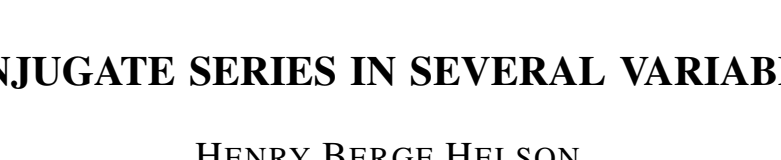




\section{CONJUGATE SERIES IN SEVERAL VARIABLES}

\section{HENRY HELSON}

1. Introduction. For any trigonometric series

$$
\sum_{-\infty}^{\infty} a_{n} e^{i n x}
$$

the conjugate series is defined to be

$$
\sum_{-\infty}^{\infty}-i a_{n} \operatorname{sgn} e^{i n x}
$$

with the convention that $s g 0=0$. A series in two variables

$$
\sum_{m, n=-\infty}^{\infty} a_{m n} e^{i(m x+n y)}
$$

can be conjugated with respect to $x$, with respect to $y$, or with respect to both variables at once. The last possibility gives the series

$$
\sum_{m, n=-\infty}^{\infty}-a_{m n} s g(m n) e^{i(m x+n y)},
$$

and this has been called $[3,5]$ the conjugate of $(1)$.

For series in one variable, there are several theorems which state that a trigonometric series belonging to some function class always has conjugate in the same, or perhaps in a different function class. These theorems lead to similar results for series in several variables conjugated with respect to one of the variables. For example, one proves very easily that

$$
\int\left|\tilde{f}^{x}\right| d \sigma \leqq A \int|f| \log ^{+}|f| d \sigma+B
$$

where $\tilde{f^{x}}$ is the function conjugate to $f$ with respect to $x, d \sigma=d \sigma(x, y)$ is invariant measure on the torus, and $A, B$ are absolute constants.

In conjugation with respect to $x$, the coefficients $a_{m n}$ of (1) are multiplied by $-i$ in the right half-plane and by $i$ in the left half-plane. Conjugation in $y$ involves the upper and lower half-planes in the same way. Any half-plane bounded by a line of rational slope can be transformed by a linear change of variables into, say, the upper half-plane, and so there is a whole family of notions of conjugacy with corresponding theorems. If, however, we divide the plane by a line of irrational slope, it is not so clear how to prove the same theorems, although the definition of conjugacy with respect to any line is at hand. It is even

Received November 21, 1958. The author is a fellow of the Alfred P. Sloan Foundation. 
possible that there are new difficulties, because a line of irrational slope has lattice points close to it on opposite sides.

Sections 2 and 3 are devoted to the extension of several classical theorems about conjugate functions (all contained in [6, Chapter 7]) to several or infinitely many variables, where conjugacy is defined with respect to any given half-space. Like Bochner, who gave the first such extension [1], we use the method of analytic functions. In another paper [2], we used this method to obtain one result of this kind, but it seems worthwhile to present other extensions in detail as well.

In (3), one can replace $\tilde{f}^{x}$ by $\tilde{f}^{x y}=\tilde{f}$, the full conjugate of $f$ defined by (2), if the right side is increased a little [3]:

$$
\int|\tilde{f}| d \sigma \leqq A \int|f|\left(\log ^{+}|f|\right)^{2} d \sigma+B
$$

This is proved by applying one-dimensional theorems twice, and obviously this method must introduce a logarithmic factor twice. It has not been settled whether the logarithm in (4) really needs to be squared. Section 4 contains a proof that $\left(\log ^{+}\right)^{2}$ cannot be replaced in (4) by $\left(\log ^{+}\right)^{2-8}$ for any $\varepsilon>0$.

Throughout the paper we use the properties of Orlicz spaces, as presented in [4, Chapter 5] or in [6] with different notation.

2. Half-spaces and measures. It is natural to consider these problems on a discrete abelian group $G$, whose elements are $M, N, \cdots$, and the compact dual group $K$ with elements $X, Y, \ldots$. The value of a character $X$ at a point $N$ will be written $e^{i N \cdot x}$.

Definition. A subset $S$ of $G$ is called a half-space if

(a) $M+N$ belongs to $S$ with $M$ and $N$

(b) For any $N$ in $G$, exactly one of these alternatives holds: $N \in S$, or $-N \in S$, or $N=0$.

If $S$ is a half-space in $G$, we can define a linear order in $G$ compatible with the group operation by defining positive elements to be those belonging to $S$. Conversely, the set of positive elements in an ordered group is a half-space. Not all discrete groups contain non-trivial halfspaces, but they exist in profusion in the group of integral lattice points of a Euclidean space, and this is the case which interests us most. For example, if $\alpha_{1}, \cdots, \alpha_{k}$ are linearly independent real numbers, then the set of points $N=\left(n_{1}, \cdots, n_{k}\right)$ satisfying

$$
n_{1} \alpha_{1}+\cdots+n_{k} \alpha_{k}>0
$$

is a half-space in the lattice group of $k$ dimensions.

Let $S$ be a fixed half-space in $G$. The continuous functions $\varphi$ on $K$ having Fourier series of the form 


$$
\mathcal{P}(X) \sim a+\sum_{S} a_{N} e^{i N \cdot X}
$$

constitute a Banach algebra $C_{S}$ in the uniform topology. Among the complex homomorphisms of $C_{S}$ is a distinguished one defined by ${ }^{1}$

$$
\hat{\varphi}\left(M_{0}\right)=\int \rho d \sigma=a .
$$

Besides, every point $X$ of $K$ determines a homomorphism

$$
\hat{\varphi}\left(M_{X}\right)=\varphi(X) .
$$

If $F$ is a function analytic on a domain of the complex plane which contains the spectrum of $\mathcal{P}$, there is an element $\psi$ of $C_{S}$ such that

$$
F(\hat{\varphi}) \equiv \hat{\psi} \text {. }
$$

We can write simply $F(\mathcal{P})=\psi$, because $\psi$ is uniquely determined by its values $\hat{\psi}(M)$. If we apply (8) to the particular homomorphisms given by (6) and (7) we obtain respectively

$$
\begin{gathered}
\int F(\mathcal{P}) d \sigma=F\left[\int \mathcal{\rho} d \sigma\right] \\
F(\varphi(X)) \equiv \psi(X)
\end{gathered}
$$

Determine a measure $\mu$ on the complex plane, depending on $\mathcal{P}$, in the following way. For every Borel set $E$ set

$$
\mu(E)=\sigma\left(\varphi^{-1}(E)\right) \text {. }
$$

Then $\mu$ is obviously a measure with the properties

$$
\left\{\begin{array}{l}
\mu(E) \geqq 0 \\
\int d \mu=1 \\
\int F(z) d \mu(z)=F(a)
\end{array}\right.
$$

( $F$ analytic on the spectrum of $\varphi$ ).

(The last property is the same as (9) above, by virtue of (11). To be analytic on the spectrum of $\mathcal{\rho}$, it is enough that $F$ be analytic on a simply connected domain containing the support of $\mu$.) A measure $\mu$ satisfying (12) will be said to represent the point $a$.)

A measure representing a given point cannot have its mass distributed over the plane in an arbitrary way. In the next section we derive several inequalities which $\mu$ must satisfy. These inequalities will be translated into statements about the relative size of the real and imaginary parts of $\varphi$, which is to say into theorems about conjugacy.

\footnotetext{
$1 d \sigma=d \sigma(X)$ is normalized Haar measure on $K$.
} 
Not all theorems about conjugate functions are related to the properties of measures in this way, but those which are can be generalized easily to the class of groups we are considering.

\section{The main theorems.}

THEOREM 1. Suppose $\mu$ represents the point 0 and has support in the strip

$$
-\frac{\pi}{2}+\varepsilon \leqq x \leqq \frac{\pi}{2}-\varepsilon
$$

Then

$$
\int e^{|y|} d \mu(z) \leqq \frac{2}{\cos \left(\frac{\pi}{2}-\varepsilon\right)}
$$

Proof. From last formula of (12) with $F(z)=e^{i z}$ we have

$$
\begin{aligned}
1 & =\int e^{i z} d \mu(z)=\int e^{-y}(\cos x+i \sin x) d \mu(z) \\
& =\int e^{-y} \cos x d \mu(x) \geqq \cos \left(\frac{\pi}{2}-\varepsilon\right) \int e^{-y} d \mu(z) .
\end{aligned}
$$

The same calculations, with $F(z)=e^{-i z}$, give

$$
1 \geqq \cos \left(\frac{\pi}{2}-\varepsilon\right) \int e^{y} d \mu(z) \text {. }
$$

Adding these inequalities gives the desired result.

Corollary. Let $\phi$ belong to $C_{S}$ and have mean value zero. Write $\varphi=u+i v$, where $u$ and $v$ are real functions. If $|u| \leqq \frac{\pi}{2}-\varepsilon$ for some positive $\varepsilon$, then

$$
\int e^{|v|} d \sigma \leqq \frac{2}{\cos \left(\frac{\pi}{2}-\varepsilon\right)}
$$

Indeed, from the definition of $\mu$ we have

$$
\int e^{|v(X)|} d \sigma(X)=\int e^{|y|} d \mu(z) \text {. }
$$

The hypothesis on $u$ implies that the support of $\mu$ lies in the strip (13), and (15) follows then from (14).

Actually the corollary is true for bounded functions $\varphi$ with Fourier 
series (5) and mean value zero; for $\rho$ can be approximated boundedly by trigonometric polynomials satisfying all the hypotheses of the corollary.

If $u$ is a real function, we shall denote by $v=T u$ that real function having mean value zero such that $u+i v$ has Fourier series (5). Tu is obviously defined if $u$ is a trigonometric polynomial, but in other cases its existence has to be shown.

Corollary. Let $u$ be a real function such that $u \log ^{+}|u|$ is summable on $K$. Then $v=T u$ exists as a summable function and

$$
\int|v| d \sigma \leqq A+B \int|u| \log ^{+}|u| d \sigma
$$

where $A$ and $B$ are absolute constants.

This corollary is the dual, in an appropriate pair of Orlicz spaces, of the first corollary. For $t \geqq 0$ define the complementary functions

$$
\begin{aligned}
& \Phi(t)=e^{t}-t-1 \\
& \Psi(t)=(t+1) \log (t+1)-t .
\end{aligned}
$$

Denote by $L_{\Phi}$ and $L_{\Psi}$ the corresponding Orlicz spaces of real functions on $K$. These spaces are paired by the functional

$$
(f, g)=\int f g d \sigma \quad\left(f \in L_{\Phi}, g \in L_{\Psi}\right),
$$

which exhibits each as a subset of the dual space of the other. It can be proved that $L_{\Phi}$ is exactly the dual of $L_{\Psi}$; and $L_{\Psi}$ is a closed subspace of the dual of $L_{\Phi}$.

Let us consider $T$ as an operator carrying real trigonometric polynomials in the uniform norm into $L_{\Phi}$. Suppose that $u$ has mean value zero, and $\|u\|_{\infty} \leqq \pi / 4$. Using the first corollary and an elementary property of the norm in $L_{\Phi}$, we have for $v=T u$

$$
\|v\|_{\Phi} \leqq \int e^{|v|} d \sigma+1 \leqq 2 \sec \frac{\pi}{4}+1 .
$$

Therefore $T$ is a bounded operator, on this subset of the continuous functions. The restriction on the mean value of $u$ is immaterial, and so $T$ can be extended to a bounded operator mapping real continuous functions in the uniform norm into $L_{\Phi}$.

The operator $T^{*}$ adjoint to $T$ carries linear functionals on $L_{\Phi}$ to signed measures on $K$. Let $u_{1}$ and $u_{2}$ be real trigonometric polynomials with mean values $a$ and $b$ respectively. Set $v_{1}=T u_{1}, v_{2}=T u_{2}$. Then 


$$
\begin{aligned}
a b= & \int\left(u_{1}+i v_{1}\right)\left(u_{2}+i v_{2}\right) d \sigma=\int\left(u_{1} u_{2}-v_{1} v_{2}\right) d \sigma \\
& +i \int\left(u_{2} v_{1}+u_{1} v_{2}\right) d \sigma .
\end{aligned}
$$

Since $a$ and $b$ are real, the last term vanishes and

$$
\int\left(T u_{1}\right) u_{2} d \sigma=\int u_{1}\left(-T u_{2}\right) d \sigma
$$

It follows, by the definition of the adjoint operation, that

$$
T^{*} u_{2}=-T u_{2} d \sigma
$$

The statement that $T^{*}$ is bounded as an operator from $L_{\Psi}$ to the space of measures is thus

$$
\int|T u| d \sigma \leqq K\|u\|_{\Psi}
$$

From the general inequality

$$
\|u\|_{\Psi} \leqq \int \Psi(|u|) d \sigma+1
$$

(16) follows at once, at least for trigonometric polynomials. The full statement of the corollary is derived by a conventional limiting process.

It is curious that the duality argument required that $S$ be a halfspace, and not merely a cone, which would have sufficed in proving Theorem 1. However, (16) holds a fortiori for the real and imaginary parts of a double power series, for example. This result follows also from the corresponding theorem in one variable.

The second corollary is a limiting case of the theorem of Bochner already referred to. The inequality

$$
\left(\int|v|^{p} d \sigma\right)^{1 / p} \leqq A_{p} \int|u| d \sigma \quad(0<p<1),
$$

which is another limiting case, was established in [2].

THEOREM 2. Let $u$ be a non-negative trigonometric polynomial with mean value $a$, and set $v=T u$. Then

$$
\int u \log ^{+} u d \sigma \leqq(a+1) \log (a+1)+\frac{\pi}{2} \int|v| d \sigma .
$$

Proof. Let $\phi=u+i v$, so that $\phi$ belongs to $C_{S}$. It was proved in [2] that the spectrum of $\mathcal{P}$ lies in the right half-plane. Therefore $(z+1) \log (z+1)$ is analytic on the spectrum of $\mathcal{P}$, and from (9) we have 


$$
\int(u+1+i v) \log (u+1+i v) d \sigma=(a+1) \log (a+1) .
$$

The right side is real. Taking the real part of the left side gives

$$
\begin{gathered}
\int(u+1) \log |u+1+i v|-v \arctan v /(u+1) d \sigma \\
=(a+1) \log (a+1) .
\end{gathered}
$$

Using the inequality

$$
0 \leqq y \arctan y / x \leqq \frac{\pi}{2}|y|
$$

we find (17) directly.

Of course (17) leads by a limiting argument to the fact that if $u$ is a real non-negative function which is summable together with $T u$, then (17) continues to hold.

Finally we remark that Theorem 2 could, like Theorem 1, be expressed in terms of a measure representing a given point. ${ }^{2}$

4. Theorem 3. Given $\varepsilon>0$, there is a function $f$ with Fourier series (1) and satisfying

$$
\int|f|\left(\log ^{+}|f|\right)^{2-\varepsilon} d \sigma<\infty
$$

such that (2) is not a Fourier series.

The proof depends on a number of fairly independent observations.

(a) If $f$ is a function of one variable with Fourier series

$$
f(x) \sim \sum_{0}^{\infty} a_{n} e^{i n x},
$$

then according to a well-known theorem of Hardy and Littlewood

$$
\sum_{0}^{\infty} \frac{\left|a_{n}\right|}{n+1} \leqq K \int|f(x)| d x
$$

Bochner has remarked that (19) leads to a similar inequality for functions to two variables:

$$
f(x, y) \sim \sum_{m, n=0}^{\infty} a_{m n} e^{i(m x+n y)}
$$

implies

${ }_{2}$ Theorem 2 ceases to be true if $S$ is the subset of the two-dimensional lattice group consisting of all $(m, n)$ with $m \geqq 0$. The reason is that the functional assigning to $\varphi$ in $C_{S}$ its mean value is not multiplicative. For the corollaries of Theorem 1 the proofs indeed require that $S$ be a half-space, but the results are still essentially true if $S$ is the set just defined. 


$$
\sum_{m, n=0}^{\infty}\left|a_{m n}\right| /(m+1)(n+1) \leqq K \int|f(x, y)| d \sigma(x, y) .
$$

(b) Suppose, contrary to the statement of the theorem, that (2) is a Fourier series whenever (18) holds, for a certain positive $\varepsilon$ which is fixed from now on. We may take $\varepsilon<1$. Then the functions, $f, \tilde{f}^{x}, \tilde{f}^{y}$, and $\tilde{f}^{x y}$ are all summable, and the same must be true for the linear combination

$$
\frac{1}{4}\left\{f+i \tilde{f}^{x}+i \tilde{f^{y}}-\tilde{f^{x y}}\right\} \sum_{m, n=1}^{\infty} a_{m n} e^{i(m x+n y)}+\frac{1}{4} \sum_{m, n=0} a_{m n} e^{i(m x+n y)} .
$$

The last sum is a Fourier series, merely because $f$ is summable, and we conclude easily that

$$
\sum_{m, n=0}^{\infty} a_{m n} e^{i(m x+n y)}
$$

is a Fourier series. It follows from (a) that

$$
\sum_{m, n=0}^{\infty}\left|a_{m n}\right| /(m+1)(n+1)<\infty
$$

whenever (1) is the Fourier series of a function $f$ satisfying (18).

(c) For non-negative $t$ define

$$
\Psi_{1}(t)=(t+1) \log ^{2-\varepsilon}(t+1) .
$$

Since $\varepsilon<1, \Psi_{1}$ is a convex function vanishing together with its derivative at the origin. Denote by $\Phi_{1}$ its complementary function. A simple computation shows that for large $t$

$$
\Phi_{1}(t)>e^{k t^{\beta}}
$$

where $k$ is an appropriately chosen positive number, and $\beta=1 /(2-\varepsilon)$.

(d) The relation (20), valid for every function of the space $L_{\Psi_{1}}$, implies that

$$
\sum_{m, n=0}^{\infty} \lambda(m) \lambda(n) e^{i \tau} m n e^{i(m x+n y)}
$$

(where the $\tau_{m n}$ are arbitrary real numbers, and $\lambda(n)=1 /(n+1)$ for $n \geqq 0$ ) is the Fourier series of a function belonging to the dual space of $L_{\Psi_{1}}$. Since $\Psi_{1}(2 t) \leqq M \Psi_{1}(t)$ for a fixed number $M$, the dual of $L_{\Psi_{1}}$ is exactly $L_{\Phi_{1}}\left[4\right.$, p. 138], so that (22) belongs to $L_{\Phi_{1}}$ for every choice of the $\tau_{m n}$. Choose constants $\tau_{m n}$ in the particular form $\tau_{m}+\tau_{n}$, where $\left\{\tau_{n}\right\}_{0}^{\infty}$ is a simple sequence of real numbers. Then (22) is formally the product of 


$$
F(x) \sim \sum_{0}^{\infty} \lambda(n) e^{i \tau} n e^{i n x}
$$

and the same series in $y$. Since (22) represents a function of two variables which is square-summable, the coefficients in (23) are square-summable and $F$ is in $L^{2}$ as a function of one variable. Moreover the function represented by (22) really is $F(x) F(y)$, as one can see in an elementary way.

(e). Lemma. Let $\alpha$ be a given positive number, and $g$ a nonnegative function on some finite measure space with measure d $\mu$, normalized to have unit total mass. A necessary and sufficient condition to have

$$
\int e^{s g^{\alpha}} d \mu<\infty \text { for some } s>0
$$

is that $g$ belong to $L^{q}$ for every finite $q$ and satisfy

$$
\|g\|_{q}=O\left(q^{1 / \alpha}\right) .
$$

In particular, $g$ belongs to $L_{\Phi}$ (where $\Phi(t)=e^{t}-t-1$ as heretofore) just if $\|g\|_{q}=O(q)$.

To prove the lemma, let $s$ be positive and consider

$$
\int e^{s g^{\alpha}} d \mu=1+\sum_{1}^{\infty} \frac{s^{n}}{n !} \int g^{n \alpha} d \mu=1+\sum_{1}^{\infty} \frac{s^{n}}{n !}\|g\|_{n \alpha}^{n \alpha} .
$$

The series converges for some $s>0$ if and only if

$$
\lim \sup \left[\frac{1}{n !}\|g\|_{n \alpha}^{n \alpha}\right]^{1 / n}=\lim \sup \frac{1}{n}\|g\|_{n \alpha}^{\infty}<\infty .
$$

Because $\|g\|_{q}$ is monotone in $q$, this means the same as

$$
\lim \sup q^{-1 / \alpha}\left\|_{q} g\right\|<\infty
$$

which was to be proved.

Now we can finish the proof of the theorem. Assuming the theorem was false for a certain positive $\varepsilon$, we deduced in (d) that $G(x, y)=$ $F(x) F(y)$ belongs to $L_{\Phi_{1}}$, where $\Phi_{1}$ satisfies (21) and $F$ is defined by (23). The lemma in (e) implies then

$$
\|G\|_{q}=O\left(q^{1 / \beta}\right)
$$

But $\|G\|_{q}=\left[\|F\|_{q}\right]^{2}$, and therefore

$$
\|F\|_{q}=O\left(q^{1 / 2 \beta}\right) .
$$


This is the crucial point of the proof. Since $\beta>1 / 2,\|F\|_{q}$ grows less rapidly than $q$, and we can show that this is false.

Indeed, from (25) and the lemma it follows that

$$
\int e^{s|F|^{2 \beta}} d x / 2 \pi<\infty
$$

for some positive $s$. Set $\Phi_{2}(t)=e^{t^{2 \beta}}-1$ for non-negative $t$, and let $\Psi_{2}$ be the complementary function. For large $t$ we have

$$
\Psi_{2}(t)<t \log ^{1 / 2 \beta} t
$$

We are interested now in the spaces $L_{\Phi_{2}}$ and $L_{\Psi_{2}}$, formed with functions defined on the circle and measure $d x / 2 \pi$. If $f$ and $g$ belong to $L_{\Phi_{2}}$ and $L_{\Psi_{2}}$ respectively, and have Fourier coefficients $\left\{a_{n}\right\}$ and $\left\{b_{n}\right\}$, then $\sum_{-\infty}^{\infty} a_{n} b_{n}$ is $(C, 1)$-summable at least $[6$, p. 88]. In particular, taking $F$ for $f$,

$$
\sum_{0}^{\infty} \frac{e^{i \tau_{n}}}{n+1} b_{n}
$$

is $(C, 1)$-summable to a finite value, no matter how the $\tau_{k}$ are chosen. Therefore

$$
\sum_{0}^{\infty} \frac{\left|b_{n}\right|}{n+1}<\infty
$$

whenever the $b_{n}$ are the Fourier coefficients of a function $g$ in $L_{\Psi_{2}}$. This inequality can be sharpened by the uniform boundedness principle to

$$
\sum_{0}^{\infty} \frac{\left|b_{n}\right|}{n+1}<A\|g\|_{\Psi_{2}}<B+C \int|g| \log ^{+1 / 2 \beta}|g| d x
$$

Choose for $g$ the function equal to $1 / 2 \delta$ on $(-\delta, \delta)$ and zero elsewhere on $(-\pi, \pi)$. For $n \geqq 1$ we have $b_{n}=\sin n \delta / 2 \pi n \delta$. For small values of $\delta$, the left side of (26) exceeds a constant multiple of $\log 1 / \delta$; but the right side is only a constant times $\log ^{1 / 23} 1 / \delta$. Since $2 \beta>1$ this is impossible, and the contradiction establishes the theorem.

A modification of the same proof will show that no function of order smaller than $t \log ^{2} t$ and having sufficiently regular growth can serve in the hypothesis of the theorem on conjugate functions.

\section{REFERENCES}

1. S. Bochner, Additive set functions on groups, Ann. of Math., 40 (1939), 769-799.

2. H. Helson, Conjugate series and a theorem of Paley, Pacific J. Math., 8 (1958), 437-446. 
3. K. Sokol-Solowski, On trigonometric series conjugate to Fourier series of two variables, Fund. Math., 33 (1939-46), 166-182.

4. A. C. Zaanen, Linear Analysis, New York, 1953.

5. A. Zygmund, On the boundary values of functions of several complex variables, I, Fund. Math., 36 (1949), 207-235.

6. A. Zygmund, Trigonometrical Series, Warsaw, 1935.

UNiversity of CALIFornia, Berkeley, CALIFornia 



\section{PACIFIC JOURNAL OF MATHEMATICS}

\section{EDITORS}

\section{David Gilbarg}

Stanford University

Stanford, California

\section{R. A. Beaumont}

University of Washington

Seattle 5 , Washington

\author{
A. L. Whiteman
}

University of Southern California Los Angeles 7, California

L. J. Paige

University of California

Los Angeles 24, California

\author{
E. F. BECKENBACH \\ C. E. BURGESS \\ E. HEWITT \\ A. HORN
}

\author{
V. GANAPATHY IYER \\ R. D. JAMES \\ M. S. KNEBELMAN \\ L. NACHBIN
}

ASSOCIATE EDITORS
I. NIVEN

T. G. OSTROM

H. L. ROYDEN

M. M. SCHIFFER
E. G. STRAUS

G. SZEKERES

F. WOLF

K. YOSIDA

\section{SUPPORTING INSTITUTIONS}

\author{
UNIVERSITY OF BRITISH COLUMBIA \\ CALIFORNIA INSTITUTE OF TECHNOLOGY \\ UNIVERSITY OF CALIFORNIA \\ MONTANA STATE UNIVERSITY \\ UNIVERSITY OF NEVADA \\ OREGON STATE COLLEGE \\ UNIVERSITY OF OREGON \\ OSAKA UNIVERSITY \\ UNIVERSITY OF SOUTHERN CALIFORNIA
}

\author{
STANFORD UNIVERSITY \\ UNIVERSITY OF TOKYO \\ UNIVERSITY OF UTAH \\ WASHINGTON STATE COLLEGE \\ UNIVERSITY OF WASHINGTON \\ * * * \\ AMERICAN MATHEMATICAL SOCIETY \\ CALIFORNIA RESEARCH CORPORATION \\ HUGHES AIRCRAFT COMPANY \\ SPACE TECHNOLOGY LABORATORIES
}

Mathematical papers intended for publication in the Pacific Journal of Mathematics should be typewritten (double spaced), and the author should keep a complete copy. Manuscripts may be sent to any one of the four editors. All other communications to the editors should be addressed to the managing editor, L. J. Paige at the University of California, Los Angeles 24, California.

50 reprints per author of each article are furnished free of charge; additional copies may be obtained at cost in multiples of 50 .

The Pacific Journal of Mathematics is published quarterly, in March, June, September, and December. The price per volume (4 numbers) is $\$ 12.00$; single issues, $\$ 3.50$. Back numbers are available. Special price to individual faculty members of supporting institutions and to individual members of the American Mathematical Society: $\$ 4.00$ per volume; single issues, $\$ 1.25$.

Subscriptions, orders for back numbers, and changes of address should be sent to Pacific Journal of Mathematics, 2120 Oxford Street, Berkeley 4, California.

Printed at Kokusai Bunken Insatsusha (International Academic Printing Co., Ltd.), No. 6, 2-chome, Fujimi-cho, Chiyoda-ku, Tokyo, Japan.

PUBLISHED BY PACIFIC JOURNAL OF MATHEMATICS, A NON-PROFIT CORPORATION

The Supporting Institutions listed above contribute to the cost of publication of this Journal, but they are not owners or publishers and have no responsibility for its content or policies. 


\section{Pacific Journal of Mathematics}

\section{Vol. 9, No. $2 \quad$ June, 1959}

Lee William Anderson, On the breadth and co-dimension of a topological lattice

Frank W. Anderson and Robert L. Blair, Characterizations of certain lattices

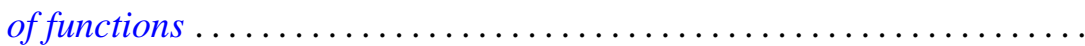

Donald Charles Benson, Extensions of a theorem of Loewner on integral

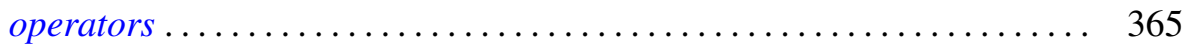

Errett Albert Bishop, A duality theorem for an arbitrary operator ........ 379

Robert McCallum Blumenthal and Ronald Kay Getoor, The asymptotic distribution of the eigenvalues for a class of Markov operators ........

Delmar L. Boyer and Elbert A. Walker, Almost locally pure Abelian

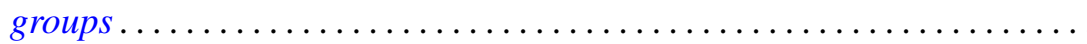

Paul Civin and Bertram Yood, Involutions on Banach algebras ........... Lincoln Kearney Durst, Exceptional real Lehmer sequences .... 415

Eldon Dyer and Allen Lowell Shields, Connectivity of topological

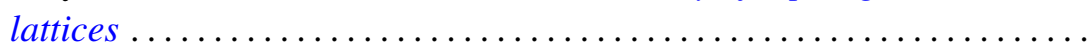

Ronald Kay Getoor, Markov operators and their associated

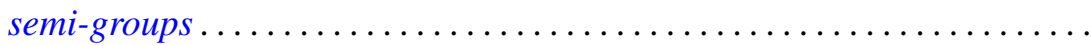

Bernard Greenspan, A bound for the orders of the components of a system of algebraic difference equations

Branko Grünbaum, On some covering and intersection properties in

Minkowski spaces ............................

Bruno Harris, Derivations of Jordan algebras ..............

Henry Berge Helson, Conjugate series in several variables.

Isidore Isaac Hirschman, Jr., A maximal problem in harmonic analysis.

II .

Alfred Horn and Robert Steinberg, Eigenvalues of the unitary part of a matrix

Edith Hirsch Luchins, On strictly semi-simple Banach algebras ...

William D. Munro, Some iterative methods for determining zeros of

functions of a complex variable...

John Rainwater, Spaces whose finest uniformity is metric .

William T. Reid, Variational aspects of generalized convex functions ....

A. Sade, Isomorphisme d'hypergroupoï des isotopes ...... . .

Isadore Manual Singer, The geometric interpretation of a special

connection . . .

Charles Andrew Swanson, Asymptotic perturbation series for characteristic

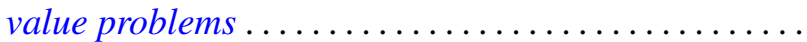

